\title{
ACTA
}

AMAZONICA

\section{Ocorrência de Centris (Hemisiella) vittata Lepeletier (Hymenoptera: Apidae: Centridini) no Cerrado s.l. do Nordeste do Maranhão, Brasil}

Marina da Costa RAMOS, Márcia Maria Correia RÊGO, Patrícia Maia Corrêa de ALBUQUERQUE

\section{RESUMO}

Fêmeas de Centris vittata Lepeletier foram observadas visitando flores de Byrsonima sp. e nidificando em troncos de Astronium sp. (Anacardiaceae) em uma área de Mata Mesofítica, em Urbano Santos (3 12’28”'S; 4324’12”), Maranhão, Brasil.

PALAVRAS-CHAVE

Abelhas solitárias, Centris vittata, abelhas de óleo, Byrsonima sp., ninhos.

\section{Occurrence of Centris (Hemisiella) vittata Lepeletier (Hymenoptera: Apidae: Centridini) in the Cerrado s.l. Bioma of Maranhão, Northeastern Brazil}

\section{ABSTRACT}

Females of Centris (Hemisiella) vittata Lepeletier were observed visiting flowers of Byrsonima sp. (Malpighiaceae) and nesting in Astronium sp. (Anacardiaceae) in a mesophytic forest area at Urbano Santos, Maranhão, Northeastern Brazil.

KEY-WORDS

Solitary bees, Centris vittata, oil bees, Byrsonima sp., nests. 
As abelhas do gênero Centris Fabricius, 1804 distribuem-se do sudoeste dos Estados Unidos até as regiōes xéricas da Argentina e Chile. Essas abelhas, solitárias, têm os mais variados hábitos de nidificação. Muitas espécies escavam ninhos no solo (Alcock et al., 1976; Batra \& Schuster, 1977; Coville et al., 1983; Vinson et al., 1993; Aguiar \& Gaglianone, 2003), outras podem estabelecer seus ninhos dentro de termiteiros (Vesey-Fitzgerald, 1939; Bennet, 1964; Gaglianone, 2001) ou em cavidades préexistentes, como troncos de árvores (Michener \& Lange, 1958; Vinson et al., 1993; Frankie et al., 1993) e ninhos armadilhas (Morato et al., 1999; Jesus \& Garófalo, 2000; Gazola \& Garófalo, 2003; Aguiar \& Garófalo, 2004).

As fêmeas da tribo Centridini são dotadas de estruturas especializadas para coleta de lipídios florais e estão associadas, principalmente às flores de Malpighiaceae (Vogel, 1988; Buchmann, 1987). Este recurso é utilizado para alimentação larval, juntamente com o pólen (Vinson et al., 1997), e também na construção das células de cria (Hiller \& Wittmann, 1994). Essa interação confere aos Centridini um papel relevante como agentes polinizadores das plantas de óleo (Frankie et al., 1983; Rêgo \& Albuquerque, 1989; Machado 2004).

No Brasil, espécies do gênero Centris foram diagnosticadas em diversos ecossistemas, dentre eles dunas e restinga (Silva \& Martins, 1999; Silva et al., 2001; Viana \& Alves-dos-Santos, 2002), caatinga (Martins, 1994; Zanella, 2000; Aguiar \& Almeida, 2002; Aguiar et al., 2003), e cerrado; neste último, com maior diversidade (Silveira \& Campos, 1995; Albuquerque \& Mendonça, 1996; Gaglianone, 2003).

Hemisiella é um grupo de ampla distribuição com a maioria das espécies ocorrendo na América do Sul. Centris (Hemisiella) vittata Lepeletier, 1841 é uma abelha de médio porte dentre os Centridini que ocorre na América do Sul e América Central. Há registros desta espécie no México, Costa Rica, Panamá e Honduras (Snelling, 1984). No Brasil há citaçōes para os Estados de São Paulo, Espírito Santo, Minas Gerais e Bahia (Silveira et al., 2002). Até então, não havia sido diagnosticada no Maranhão (Rebêlo et al., 2003). Esta é a primeira citação para o Estado.

Fêmeas de Centris (Hemisiella) vittata Lepeletier foram observadas em visita às flores do "murici" Byrsonima sp. e nidificando em cavidades pré-existentes em troncos mortos de aroeira, Astronium sp. (Anacardiaceae), expostos ao sol durante quase todo o dia. O sítio de observação, na Reserva Santo Amaro (3 12' 28"S; 4324'12"W), Município de Urbano Santos, Nordeste do Maranhão encontra-se sob o domínio de fragmentos de cerrado, entretanto os ninhos amostrados foram localizados em uma área aberta de Mata Mesofítica distante aproximadamente $300 \mathrm{~m}$ da Mata Ciliar, na mesma localidade. Segundo Frankie et al. (1993), C. vittata é uma espécie restrita a ambientes úmidos, de matas.

Os indivíduos, machos e fêmeas, que emergiram dos ninhos foram coletados. Os exemplares encontram-se depositados na coleção de referência do Laboratório de Estudos sobre Abelhas (LEA) do Departamento de Biologia da Universidade Federal do Maranhão.

\section{AGRADECIMENTOS}

Ao Conselho Nacional de Pesquisa e Desenvolvimento Científico e Tecnológico (CNPq), pela concessão da Bolsa de Iniciação Científica ao primeiro autor; à Comercial \& Agrícola Paineiras, pelo apoio logístico em campo; ao biólogo Adriano Oliveira Maciel, pela ajuda em campo; ao Prof. Fernando Zanella, do Departamento de Engenharia Florestal da Universidade de Campina Grande, pela confirmação da identificação da espécie de abelha; ao Profo Nivaldo Figueiredo, do Departamento de Biologia da Universidade Federal do Maranhão, pela identificação da aroeira.

\section{BIBLIOGRAFIA CITADA}

Aguiar, C.M.L.; Almeida, G.F. 2002. Atividade diária de coleta de óleo por espécies de Centris (Hymenoptera: Apidae) em Mcvaughia bahiana W.R. Anderson (Malpighiaceae) na caatinga. Acta. Biol. Leop., 24 (2): 131-140.

Aguiar, C.M.L.; Gaglianone, M.C. 2003. Nesting biology of Centris (Centris) aenea Lepeletier (Hymenoptera, Apidae, Centridini). Rev. Bras. Zool., 20 (4): 601-606.

Aguiar, C.M.L.; Zanella, F.C.V.; Martins, C.F.; Carvalho, C.A.L. 2003. Plantas visitadas por Centris spp. (Hymenoptera: Apidae) na Caatinga para obtenção de recursos florais. Neotropical Entomology, 32 (2): 247-259.

Aguiar, C.M.L.; Garófalo, C.A. 2004. Nesting biology of Centris (Hemisiella) tarsata Smith (Hymenoptera, Apidae, Centridini). Rev. Bras. Zool., 21 (3): 477-486.

Albuquerque, P.M.C.; Mendonça, J.A.C. 1996. Anthophoridae (Hymenoptera, Apoidea) e Flora associada em uma formação de cerrado no município de Barreirinhas, Ma, Brasil. Acta Amazônica, 26(1/2): 45-54.

Alcock, J.; Jones, C.E.; Buchmann, S.L. 1976. The nesting behavior of three species of Centris bees (Anthophoridae: Hymenoptera). J. Kansas Entomol. Soc., 49 (4): 469-474.

Batra, S.W.T.; Schuster, J.C. 1977. Nests of Centris, Melissodes, and Colletes in Guatemala (Hymenoptera: Apoidea). Biotropica, 9 (2): 135-138.

Bennett, F.D. 1964. Notes on the nesting of Centris derasa. PanPacif. Ent., 40 (2): 125-128.

Buchmann, S.L. 1987. The ecology of oil flowers and their bees. Ann. Rev. Ecol. Syst., 18: 343-369.

Coville, R.E.; Frankie, G.W.; Vinson, S.B. 1983. Nests of Centris segregata. (Hymenoptera: Anthophoridae) with a Review of the Nesting Habitats of the Genus. J. Kansas Entomol. Soc., 56(2): 109-122.

Fabricius, J.C. 1804. Systema piezatorum. Carolum Reichard, Brunsviga. 439pp. 
Frankie, G.W.; Haber, W.A.; Opler, P.A.; Bawa, K.S. 1983. Characteristics and organization of the large bee pollination system in the Costa Rican dry forest, p. 411-447. In: Jones, C.E.; Little, R.J. (eds.), Handbook of Experimental Pollination Biology. Nova York, Van Nostrand Reinhold Co. 558pp.

Frankie, G.W.; Newstrom, L.; Vinson, S.B.; Barthell, J.F. 1993. Nesting-habitat preferences of selected Centris bee species in Costa Rican dry Forest. Biotropica, 25 (3): 322-333.

Gaglianone, M.C. 2001. Nidificação e forrageamento de Centris (Ptilotopus) scopipes Friese (Hymenoptera, Apidae). Rev. Bras. Zool., 18(Supl.1): 107-117.

Gaglianone, M.C. 2003. Abelhas da tribo Centridini na Estação Ecológica de Jataí (Luís Antonio, SP): composição de espécies e interaçōes com flores de Malpighiaceae, p.279-284. In: Melo, G.A.R.; Santos, I.A. (eds.), Apoidea Neotropica: Homenagem aos 90 anos de Jesus Santiago Moure, Criciúma: UNESC. 320pp.

Gazola, A.L.; Garófalo, C.A. 2003. Parasitic behavior of Leucospis cayennensis Westwood (Hymenoptera, Leucospidae) and rates of parasitism in populations of Centris (Heterocentris) analis (Fabricius) (Hymenoptera: Apidae: Centridini). J. Kansas Entomol. Soc., 76(2): 131-142.

Hiller, B.; Wittmann, D. 1994. Seasonality, nesting biology and mating behavior of the oil-collecting bee Epicharis dejeanii (Anthophoridae, Centridini). Biociências, 2(1): 107-124.

Jesus, B.M.V.; Garófalo, C.A. 2000. Nesting behaviour of Centris (Heterocentris) analis (Fabricius) in southeastern Brazil (Hymenoptera, Apidae, Centridini). Apidologie, 31 (4): 503-515.

Lepeletier de Saint-Fargeau, A.L.M. 1841. Histoire naturelle des insectes - Hyménoptères. Librarie Encyclopédique de Roret, Paris. 680pp.

Machado, I.C. 2004. Oil collecting bees and related plants: a review of the studies in the last twenty years and case histories of plants occurring in NE Brazil, pp 255-279. In: Freitas, B. M., coord. Solitary bees: conservation, rearing and management for pollination. Fortaleza, Imprensa Universitária. 285pp.

Martins, C.F. 1994. Comunidade de abelhas (Hym., Apoidea) da caatinga e do cerrado com elementos de campo rupestre do Estado da Bahia, Brasil. Rev. Nordestina Biol., 9(2): 225-257.

Michener, C.; Lange, R.B. 1958. Observations on the ethology of neotropical Anthophorine bees (Hymenoptera: Apoidea). Univ. Kansas Sci. Bull., 39 (3): 285-291.

Morato, E.; Garcia, M.V.B.; Campos, L.A.O. 1999. Biologia de Centris Fabricius (Hymenoptera, Anthophoridae, Centridini) em matas contínuas e fragmentos na Amazônia Central. Rev. Bras. Zool., 16(4): 1213-1222.

Rebêlo, J.M.M.; Rêgo, M.M.C.; Albuquerque, P.M.C. 2003. Abelhas (Hymenoptera, Apoidea) da região setentrional do Estado do Maranhão, Brasil. In: Melo, G.A.R.; Santos, I.A. (eds.), Apoidea Neotropica: Homenagem aos 90 anos de Jesus Santiago Moure, Criciúma: UNESC. p. 265-278.
Rêgo, M.M.C.; Albuquerque, P.M.C. 1989. Comportamento das abelhas visitantes de murici, Byrsonima crassifolia (L.) Kunth, Malpighiaceae. Bol. Mus. Para. Emílio Goeldi, sér. Zool., 5 (2):179193.

Silva, M.C.M.; Martins, C. 1999. Flora apícola e relaçōes tróficas de abelhas (Hymenoptera: Apoidea) em uma área de duna (praia de Interlagos, Cabedelo-PB, Brasil). Principia, 7(3): 40-51.

Silva, F.O.; Viana, B.F.; Neves, E.L. 2001. Biologia e Arquitetura de ninhos de Centris (Hemisiella) tarsata Smith (Hymenoptera: Apidae: Centridini). Neotropical Entomology, 30(4): 541-545.

Silveira, F.A.; Campos, M.J.O. 1995. A mellissofauna de Corumbataí (SP) e Paraopeba (MG) e uma análise da biogeografia das abelhas do cerrado brasileiro (Hymenoptera, Apoidea). Rev. Bras. Entomol., 39(2): 371-401.

Silveira, F.A.; Melo, G.A.R.; Almeida, E.A.B. 2002. Abelhas brasileiras. Sistemática e Identificação. Fundação Araucária, Belo Horizonte. 253pp.

Snelling, R.S. 1984. Studies on the taxonomy and distribuition of American centridine bees (Hymenoptera: Anthophoridae). Contrib. Science, Natural History Museum of Los Angeles County, 347. p. 46.

Vesey-Fitzgerald, D. 1939. Observations on bees in Trinidad, B.W. I. Proc. Royal Ent. Soc. London., (A) 14: 107-110.

Viana, B.F.; Alves-dos-Santos, I. 2002. Bee diversity of the coastal sand dunes of Brazil, p. 135-153. In: P.G. Kevan; V.L. ImperatrizFonseca (eds.), Pollinating bees: The conservation link between agriculture and nature. Ministério do Meio Ambiente, Brasília, 313pp.

Vinson, S.B.; Frankie, G.W.; Barthell, J. 1993. Threats to the diversity of solitary bees in a Neotropical Dry forest in Central America p. 53-81. In: LaSalle, J.; Gauld, I.D. (eds) Hymenoptera and Biodiversity London: C.A.B. International. 348pp.

Vinson, S.B.; William, H.J.; Frankie, G.W.; Shrum, G. 1997. Floral lipid chemistry of Byrsonima crasssifolia (Malpighiaceae) and a use of floral lipids by Centris bees (Hymenoptera: Apidae) Biotropica, 29(1):76-83.

Vogel, S. 1988. Die Ölblumen-Symbiosen: Parallelismus und andere Aspekte ihrer Entwicklung in Raum and Zeit. Z. Zool. Syst. Evolutionsforsch 26: 341-362.

Zanella, F.C.V. 2000. The bees of the Caatinga (Hymenoptera, Apoidea, Apiformes): a species list and comparative notes regarding their distribution. Apidologie, 31(5): 579-592.

Recebido em 07/03/2006

Aceito em 01/03/2007 
